\title{
Thermoelastic and thermoplastic effects during loading and unloading of an austenitic steel
}

\author{
by E. A. Pieczyska, S. P. Gadaj and W. K. Nowacki \\ Institute of Fundamental Technological Research, PAS, 21 Swiętokrzyska St., 00-049 Warsaw, \\ Poland
}

\begin{abstract}
The effects of thermomechanical coupling occurring in metal during consecutive tensile tests were examined. The relations between temperature and stress and strain changes were investigated both in elastic and plastic ranges. The change of the character of the sample temperature was applied as a criterion for the limit between the elastic and plastic regime. The thermomechanical coupling effects were interpreted in terms of the material microstructure evolution. The thermal effects concomitant with the thermoelastic unloading were evaluated.
\end{abstract}

\section{Introduction}

Effects of thermomechanical coupling which occur frequently in nature have a long history both in theoretical and experimental work [1-3]. The thermomechanical coupling in materials of regular structure (e.g. in metals) can be classified according to their initial state [4].

The effects of thermomechanical coupling in ideal crystals without any lattice defects are related to the pressure and volume change of the body under examination:

$$
\pm \Delta p \rightarrow \pm \Delta T \text { or } \pm \Delta V \rightarrow \pm \Delta T
$$

where: $p$-pressure, $V$-volume and $T$ - absolute temperature.

The temperature changes can be positive, negative or equal zero according to the kind of deformation (compression, elongation, shear, torsion). These temperature changes are first of all concomitant with initial reversible deformation ranges to a significant extent. Their contribution to the further deformation range becomes less significant due to the predominant weight of durable plastic deformation.

In "normal" defected material, usually at some extent of deformation, the thermomechanical coupling occurs as a result of the material microstructure change related to its plastic deformation. This process takes place due to generation, motion, and annihilation of crystal lattice defects, mainly dislocations. The changes of the density and configuration of the defects always lead to the energy dissipation, which causes the temperature increase. These effects can be significant and quantitatively they are not comparable with the effects of thermomechanical coupling related to pressure or volume change, which usually does not exceed a fraction of $K$.

The definite irreversible plastic deformation is preceded by the reversible elastic one in the most commonly used materials.

The temperature change of a sample subjected to adiabatic uniaxial elastic deformation related to the pure volumetric deformation and called the thermoelastic (piezocalorimetric) effect can be derived from the first and the second law of thermodynamics $[1,5]$.

Assuming that the coefficients of the material used in the Kelvin (Thomson) formula are constant, the elastic deformation is described by the linear dependence between the temperature and the stress change:

$$
\Delta T_{e l}=-k \times \Delta \sigma_{s} .
$$


The temperature characteristic deviation from the straight line indicates the change in the nature of the process; it runs through the pure elastic to the elasto-plastic deformation $[3,6]$.

The investigation of both thermoelastic and thermoplastic effects concomitant with loading and unloading of the sample subjected to consecutive tensile deformation is the subject of this paper. The outset of plastic deformation evolving in the subsequent cycles of loading is examined this way. The evaluated values of the thermomechanical coupling effects are interpreted in terms of the material microstructure evolution under tensile deformation and referred to its strain hardening degree. The evaluation of the thermal effects concomitant with the material thermoelastic unloading is of interest.

\section{Experimental details}

The sheet specimens of austenitic steel were subjected to elongation by means of a testing machine with a constant rate of loading and unloading, equal to $2 \times 10-3 \mathrm{~s}-1$ (strain rate). The gauge length was $25 \pm 5 \mathrm{~mm}$. The samples were made from cold-rolled steel strips of $25 \mathrm{~mm} \times 2 \mathrm{~mm}$ cross-section, annealed at $1050 \mathrm{~K}$ for $40 \mathrm{~min}$. The shape and dimensions of the specimen were detailed previously [6]. The grain size was about $50 \mu \mathrm{m}$. Testes were performed in the following way: - loading up to $4 \%$ of deformation, unloading, cooling the specimen to its initial temperature, and straining to $4 \%$ again. Five such cycles - loading and unloading for each sample - were performed. The stress versus strain, the stress, and the strain versus time were continuously recorded. The distribution of infrared radiation emitted by the sample surface was monitored using the thermovision camera AGA - 680 interconnected with a computer system. The infrared radiation, registered by the thermovision equipment, allows us to obtain the temperature evolution of the sample with high precision, up to $0.02 \mathrm{~K}$ [2]. In each cycle of straining, thermoelastic effects, i.e. the decrease in temperature in the initial stage of elongation and the increase in temperature during the thermoelastic unloading were examined.

\section{Results and discussion}

The mechanical characteristics and the distributions of intensity of infrared radiation allow us to obtain the stress-strain relations (Fig. 1) and the temperature evolution of the specimen subjected to the consecutive loading cycles (Fig. 2).

A clearly seen yield point appears in the course of the strain hardening and it becomes more pronounced in the subsequent cycles of deformation (Fig. 1).

At the beginning of each cycle in the elastic stage of elongation, a temperature decrease is observed that is followed by a temperature increase that is typical for the plastic deformation (Fig. 2). The process of unloading is again accompanied by an increase in temperature. This seemingly strange result can be explained on the base of analysis of the thermoelastic unloading.

At the moment of onset of unloading (indicated by $x$ in Fig. 2), the direction of the loading changes. Then a negative increment of stress occurs in the material still being subjected to tension. Such negative stress increments induce positive increments in temperature, called the thermoelastic effect, similarly as in elastic compression. The increase in temperature is observed during the whole range of the thermoelastic unloading.

The middle parts of characteristics describing the plastic straining are almost straight lines with both the length and the slopes of inclination increasing in the subsequent cycles of straining. This indicates the more intensive heat emission related to the more advanced state of plastic deformation.

The temperature evolution of the sample obtained during five complete cycles of loading and unloading plotted versus stress is shown in Fig. 3: The initial parts of deformation, found for each cycle, are located on the same straight line describing the elastic deformation of the specimen and characterising its material. The point of the deviation from the straight line 
$\Delta T=-k \Delta \sigma$ (describing the elastic deformation) from the plot of measured temperature of the specimen indicates where the plastic deformation starts. The stress value corresponding to this point $\left(\sigma_{P / T}\right)$ is usually below $\sigma_{0.02}$.

The plastic deformation is featured by the increasing part of the characteristics. The rate of this increase depends on the extent of the plastic deformation, it is higher in the subsequent cycles. Furthermore, some inflection points appear on the plastic parts of the characteristics. They can be attributed to the "overstresses" observed on $\sigma(\varepsilon)$ curves in the consecutive cycles obtained for more advanced state of the material strain hardening (Fig. 1). This confirms the dislocation character of the overstresses.

During the thermoelastic unloading, temperature rise is observed again. Moreover, the curves of $\Delta T_{e l}$ un (unloading) versus stress relations obtained for various cycles are parallel to one another. They can be described by the relation $\Delta T_{e l}$ un $=-k \Delta \sigma$ (where $\Delta \sigma<0$ ) and remain almost parallel to the straight line describing the initial elastic deformation of the material: $\Delta \mathrm{T}_{\mathrm{ell} \text { load }}=-\mathrm{k} \Delta \sigma$, where $\Delta \sigma>0$.

The slope $k$ as determined from Fig. 1 is equal to $1.23 \times 10^{-3} \mathrm{Kmm}^{2} / \mathrm{N}$. The coefficient $k$, calculated on the basis of the Thomson formula (eq.1) is equal to $1.25 \times 10^{-3} \mathrm{Kmm}^{2} / \mathrm{N}$. These results agree with theory.

The first characteristics found for the initial state of material which is showing smooth transition from the elastic to plastic deformation (Fig. 3), differs from the other characteristics. During a significant part of the plastic deformation, it still displays a smooth parabolic dependence between the changes in temperature and stress related to the high rate of material hardening in this range of straining. The microstructure that develops at the start of plastic deformation creates a pattern of dislocations which systematically accommodates the material to the growing load. This accommodation entails the creation of new dislocations and the interaction of dislocations with grain boundaries, vacancies, foreign atoms etc. Dislocation mechanisms initially occur in proper (weaker) points of favourably oriented grains and lead to the activation of "easy" dislocation sources in the primary and then, in the secondary slip system.

The process of elasto-plastic transition and further plastic deformation in other cycles proceeded in a similar way. It was caused by the development of secondary slip systems and the creation of evolved dislocation structures that were initiated at the end of the first cycle of straining.

\section{Conclusions}

Both the thermoelastic and thermoplastic effects become more significant, and moreover, their course evolves as the metal hardens in the subsequent cycles of straining.

As the macroscopic irreversible plastic deformation begins when the adiabatic dependence $\Delta T(\sigma)$ is no longer linear, this point indicates the beginning of plastic deformation.

For materials without a pronounced yield limit, the method based upon the temperature measurement detects the outset of plastic strain earlier than with methods based upon the conventional limit stress derived from the $\sigma(\varepsilon)$ curve.

Considering $\Delta T(\sigma)$ curves, one can observe that similarly to the shapes observed on $\sigma(\varepsilon)$ curves. The deviation from the straight line describing the elastic deformation and characterizing the material becomes more abrupt in the following cycles.

The parts of characteristics $\Delta T(\sigma)$ describing the thermoelastic unloading are parallel to one another and remain almost parallel to the straight line describing the initial elastic deformation and characterizing the material of the specimen.

Only the results obtained for the initial undeformed state of material shows a smooth parabolic transition from the elastic to plastic deformations, observable both in mechanical, thermal and energy stored characteristics. The process of elasto-plastic transition and further plastic deformation in other cycles proceeded in a similar way. It was caused by the 
development of secondary slip systems that were initiated at the end of the first cycle of straining.

\section{REFERENCES}

[1] BEVER (M. B.), HOLT (D. L.), TITCHENER (A. L.) - The Stored Energy of Cold Work, Progress in Materials Science 17, (Pergamon Press, New York, 1973).

[2] GADAJ (S. P.), NOWACKI (W. K.), PIECZYSKA (E. A.) - Changes of temperature during the simple shear test [3] of stainless steel, Arch. Mech., 48, 4, 1996, p. 779-788.

[3] GADAJ (S. P.), NOWACKI (W. K.), PIECZYSKA (E. A.) - Investigation of Temperature Distribution during Plastic Deformation of Seminar: QIRT'96, Stuttgart, September 2-5, 1996.

[4] KLEPACZKO (J. R.) - Thermomechanical Coupling in Metals, IFTR Reports, 1978.

[5] KELVIN (L.) - On the thermoelastic and thermo-magnetic properties of matter, Trans. Roy. Soc. Edinb. 20, 161, 1853, p. 57-77.

[6] PIECZYSKA (E.) - Critical Point of the Elastoplastic Transition in terms of Thermomechanical Coupling, J. Theor. Appl. Mech., 1996, p. 281-306.

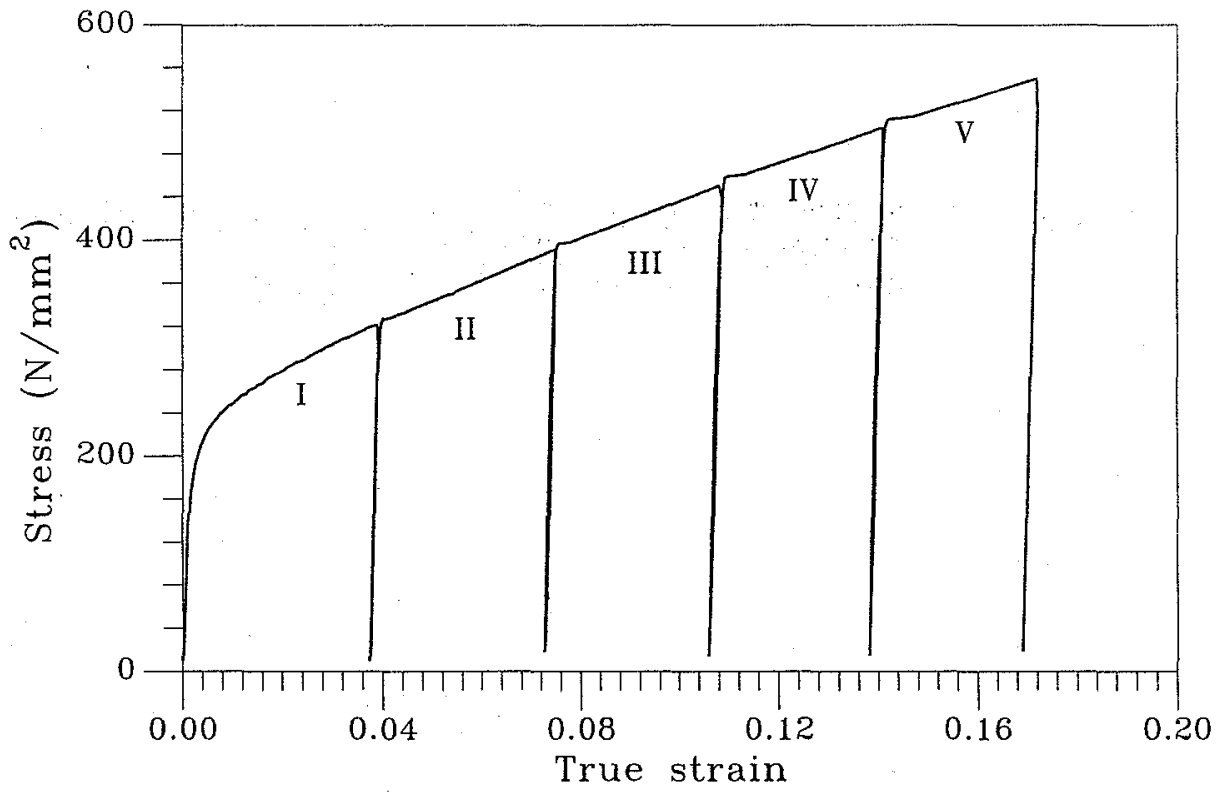

Fig. 1. Dependence of stress versus strain during consecutive cycles of loading and unloading of specimen of stainless steel 
http://dx.doi.org/10.21611/qirt.1998.017

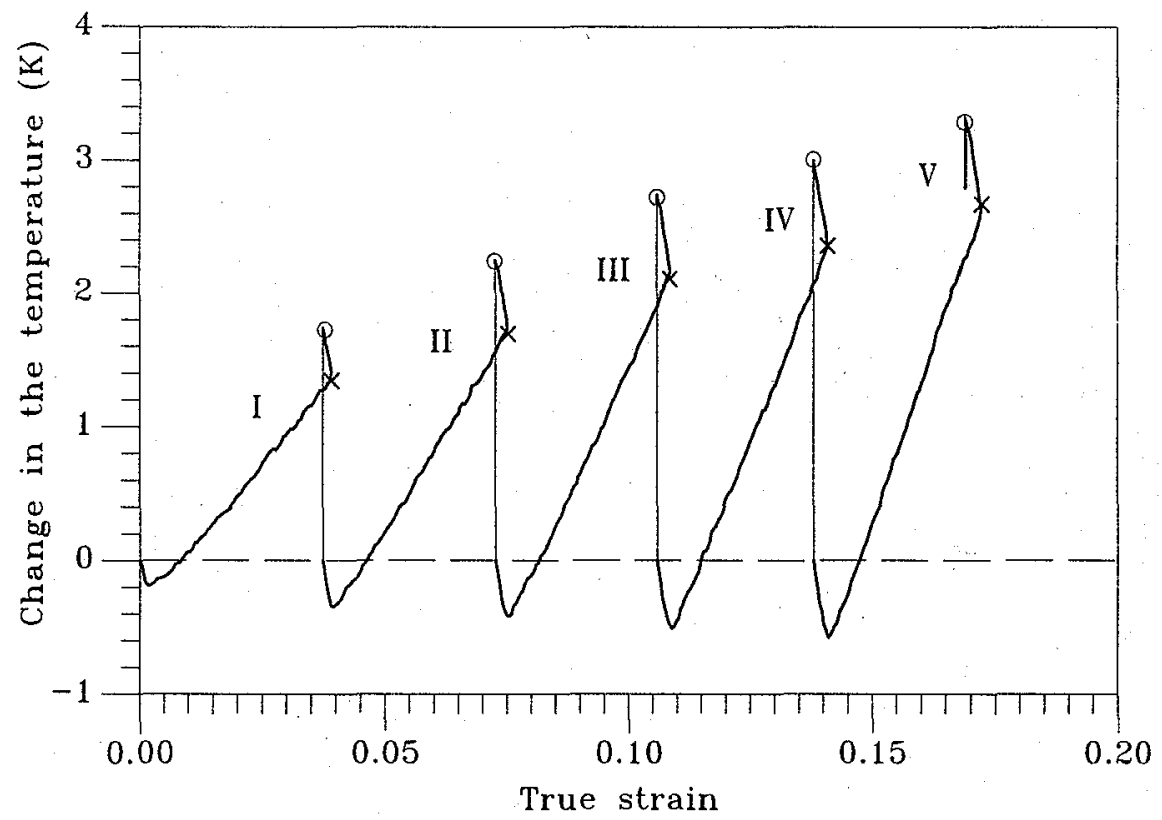

Fig. 2. Temperature changes versus deformation during successive cycles of loading and unloading of specimen of stainless steel $x$ - onset of unloading (OU), o-end of unloading (EU)

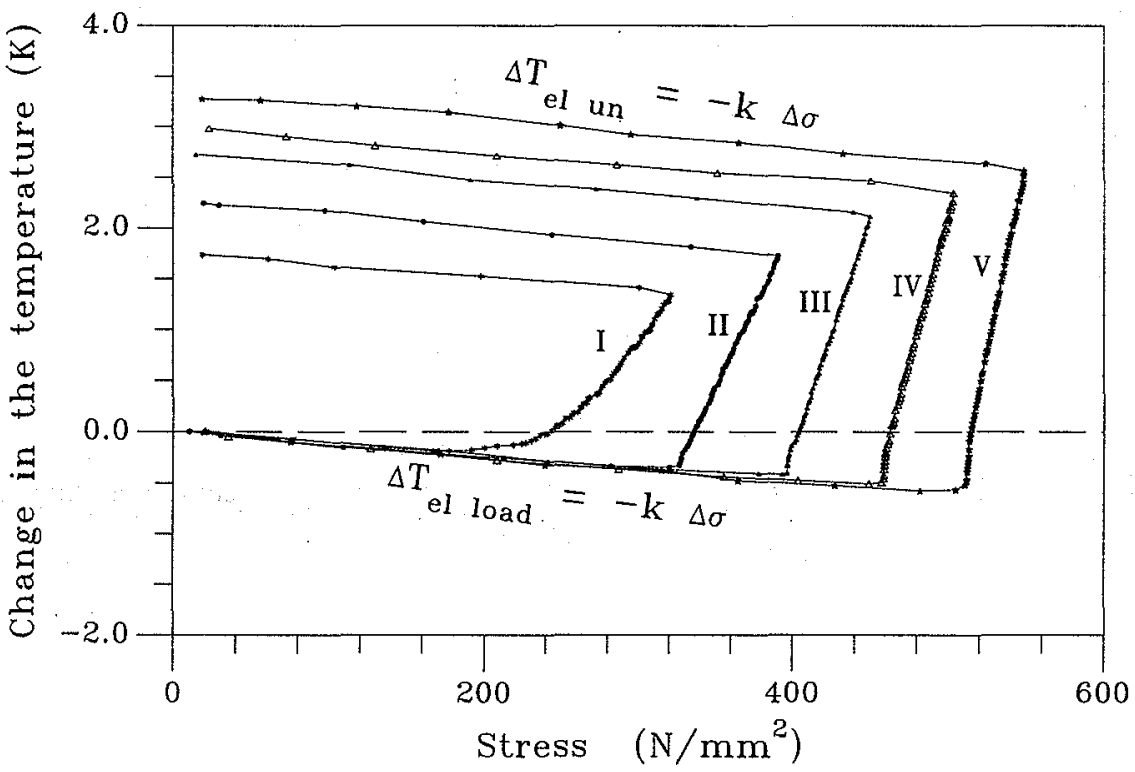

Fig. 3. Temperature changes of a stainless steel sample subjected to successive cycles of loading $(\Delta \sigma>0 \Rightarrow \Delta T<0)$ and unloading $(\Delta \sigma<0 \Rightarrow \Delta T>0)$ plotted as a function of stress 